\title{
Correction of Geometric Influence in Permittivity Determination
}

\author{
Xiangdong Xu, Tord Bengtsson*, Jörgen Blennow and Stanislaw M. Gubanski \\ Department of Materials and Manufacturing Technology \\ Chalmers University of Technology, SE 41296 Göteborg, Sweden \\ * also with ABB Corporate Research, SE 72178 Västerås, Sweden
}

\begin{abstract}
Determination of relative permittivity of dielectric materials is generally done with a rather low accuracy, in the order of several percent. This is in a sharp contrast to the accuracy of measurements of the dissipation factor, both usually being determined in the same measurement. A common understanding for the inferior accuracy in permittivity measurements is the effects of electrode edges. However, further studies indicate that geometric effects, arising from electrode shielding box, guard ring, electrode supporting materials, etc., also influence the accuracy significantly if the responding voltage present at the measuring electrode is non-negligible. With help of the Finite Element Method (FEM), geometric correction factors are estimated from an electrode model to increase the accuracy. This study is specially focused on the application of contact-free electrode arrangement using the air reference method. In this paper, a few examples of how geometric influences affect results are presented as well as a comparison of experimental results. From these insights, we discuss how to minimize and compensate the geometric effects.
\end{abstract}

\section{Introduction}

Permittivity is a key factor in selection and development of dielectric materials for various electrical apparatus constructions. It is often determined together with loss factor by applying dielectric response measurements in the frequency domain, known as frequency domain spectroscopy (FDS) [1] which is an important technique for both dielectric characterization and assessment of insulation system condition.

The accuracy of permittivity determination in dielectric material specimens is generally low, in the order of several percent. This is a sharp contrast to the accuracy of the loss factor, both of which are often determined in the same measurement. High accuracy of loss factor determination, in the range of $10^{-5}$, can be realized by AWIS technique [2] in application of contact-free electrode arrangement using air reference method [3].

The general understanding of the high uncertainty in permittivity determinations is that it is due to the electrode edge effect, distortions of the electric field lines at the electrode edge. This is a known issue in capacitance measurements. Researchers were struggling with accuracy in capacitance determinations for more than a century [4-6]. In general, there are two suggested methods to limit this effect, one is to use a guard ring which confines the effective measured area [7]; another one is to compensate the measured capacitance with a correction factor by either analytical calculations or numerical estimations $[6,8]$.

Dielectric response, in application of high voltage engineering, is often measured from a high impedance current shunt [3] and resulting in non-negligible voltages generated at the measuring electrode. With voltages present at the electrode, the measured capacitances will be larger than the capacitance between the two electrodes as the surrounding objects, such as shielding box, electrode supporting materials, connection cables etc., will have capacitive couplings with the measuring electrode and result in geometric influences.

In this paper, the finite element method (FEM) is employed to estimate a correction factor from an electrode setup model to improve the accuracy of permittivity determination. This study is specially focused on the application of contact-free electrode arrangement using the air reference method, as these techniques enable fast and accurate dielectric loss determination.

The implemented model was first used for sensitivity analysis by modifying different model parameters. Further, measurements of several layers of PET films as well as a polycarbonate specimen are used as examples to illustrate the use of correction factors.

\section{Geometric effects}

In addition to electrode edge effect, in most high voltage applications, a high impedance shunt is employed to measure the response current and thus a non-negligible voltage is generated and presented at the electrode. Consequently, the capacitive couplings between the electrode and surrounding objects are introduced; these are further enhanced by presence of electrode supporting materials in-between. As a result, the accuracy of the determined permittivity is degraded.

\subsection{Edge effect}

The electrode edge effect is the most commonly considered error in permittivity measurements. This effect appears as a result of distortions of electric field lines at the electrode edges. It will be further pronounced in contact-free electrode arrangements, as electrostatic flux at electrode edge will curve into the air layer and distort the measured specimen area.

A usual method to avoid the edge effect is to use a grounded guard-ring electrode, as illustrated in Fig.1, 


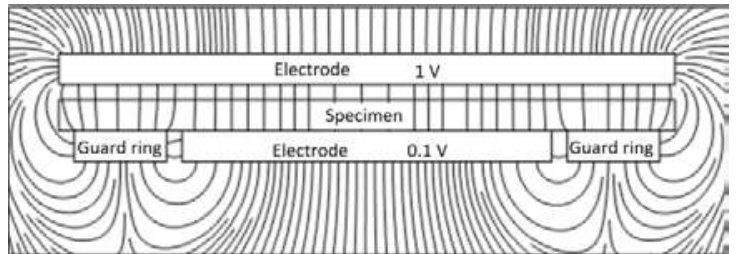

Fig. 1 - Electric field distribution of two parallel disk electrodes with guard ring, the ring electrode is grounded.

which controls the electric field and better defines the effective measurement area. This solution has been employed in many dielectric studies and is recommended by the IEC standard [1], however, it cannot eliminate the interference from the shielding box as well as from the guard-ring itself when a nonnegligible voltage is generated on the electrode by a high impedance shunt. To illustrate this effect in the figure, $0.1 \mathrm{~V}$ is applied to the bottom measuring electrode.

\subsection{Shielding box}

Another inherent error source is the capacitance between electrodes and the grounded shielding box due to the electric field lines which will pass out in all directions. The amount of capacitance increase due to this effect depends on the electrode setup and the voltage potential on other objects within the box.

Historically, the shielding box effect has been largely forgotten. One reason might be that if an operation amplifier is employed to obtain the dielectric responding current, voltage potential drop over the amplifier is considered negligible and interference from the grounded surroundings should therefore be insignificant. In applications within high voltage engineering and with interests to obtain a board highfrequency response, a high impedance capacitive shunt is required. Thus, the shielding box needs to be compensated for.

\subsection{Other influences}

As the electric field lines will pass out to all directions to earth from the electrodes when voltage is applied, the measured capacitance will be increased as discussed above. This capacitance can be even further increased if the flux passes through electrode supporting materials, such as glass, wood, plastic, etc. Apart from the supporting materials, connection cables and surroundings all add undesired capacitance. These interferences may not be neglected when high precision estimates of permittivity are desired.

\section{Correction of geometric influences}

To estimate the correction factors for the geometric influences, the same principle as used in the air reference measurement is employed. The detailed FEM model as well as the correction procedure for the air reference measurement is discussed in the following.

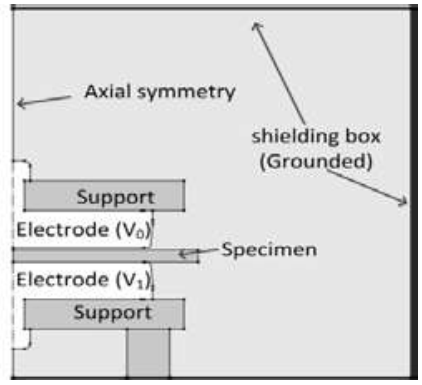

Fig. 2 - 2D symmetric finite element model for geometric influence correction in permittivity determination

\subsection{Finite element model}

To compensate for the effects from both electrode edges and surroundings, a finite-element model of the contactfree electrode setup is developed. The model was built in Comsol Multiphysics 4.2a [9] and stationary electrostatic studies were carried out to estimate the capacitances. As the accuracy of FEM calculation is rather high, the accuracy of a correction factor is limited to how complicated a model one can implement. To achieve a high precision, the model might need to be built in $3 \mathrm{D}$ with every fine detail reproduced. With increasing complexity of model configuration, the model design and computing time will also increase progressively. It was thus decided to limit this study to a 2D configuration at the present stage.

The computational domain of the considered case is illustrated in Fig. 2. The voltage potential on the electrodes are defined by initial calculations; the test gap distances as well as the thickness of specimen are adjustable. The 2D model is verified against measurements at different gap distances with air and a good agreement is achieved by adjusting the size of shielding box.

\subsection{Calculation of correction factor}

The geometric correction factor is obtained from two permittivities, the implemented specimen permittivity in the model and the calculated, "observed", permittivity. To calculate the observed permittivity from the model, the capacitance between the measuring electrode and the rest of the cell is calculated.

The voltage $\left(\mathrm{V}_{1}\right)$ generated at the measuring electrode is calculated based on a simple circuit model. It consists of three in series connected components: $\mathrm{C}_{\text {air }}, \mathrm{C}_{\text {specimen }}$, and $\mathrm{Z}_{\text {shunt }} . \mathrm{C}_{\text {air }}$ is the capacitance of the air gap above the specimen and $\mathrm{C}_{\text {specimen }}$ is specimen capacitance. Both capacitances are calculated assuming the same surface area as the measuring electrode. The applied voltage $V_{0}$ and the shunt impedance $Z_{\text {shunt }}$ are same as in the measurements. As a result, the calculated voltage potential $\mathrm{V}_{1}$ is specified for different specimenelectrode dimensions as well as specimen permittivity.

In practice, a fixed test gap distance or a fixed specimen thickness is often desired. This enables combining the test gap distance and the specimen thickness to a distance ratio $d_{r}$. The obtained correction factors are, 
therefore, only applicable for a specified test gap distance or a specified sample dimension.

To study electrostatic fields as well as electric charges in the model, steady-state electrostatics studies are carried out. In the finite element model, capacitance can be estimated from equation 3.1, where the $V_{10}$ is the voltage difference between the two electrodes $\left(V_{1}-V_{0}\right)$ and $Q$ is the total surface charge at the measuring electrode.

$$
C=\frac{Q}{V_{10}}
$$

The total surface charge $Q$ is calculated by a surface integral of the surface charge density $\rho_{s}$ at the measuring electrode:

$$
Q=\oiint_{s} \rho_{s} d s
$$

Some additional steps are required to calculate a geometric correction factor for the air reference method. Two capacitances are obtained from the model, with and without a specimen with permittivity $\left(\varepsilon_{i}\right)$ between the electrodes. The observed permittivity $\left(\varepsilon_{m}\right)$ of the specimen is then obtained by the air reference calculations [3]. The ratio of $\varepsilon_{i}$ and $\varepsilon_{m}$ is the geometric correction factor for this specific electrode-specimen arrangement.

\subsection{Model sensitivity analysis}

It is valuable to assess how the model parameters, such as voltage potentials, shielding box, supports, etc., influence the correction factor. To evaluate it, a reference model, shown in Fig. 2, is employed. The test gap is set at $1.4 \mathrm{~mm}$ and the specimen thickness is $1 \mathrm{~mm}$. The relative permittivity for the specimen and the supporting material is selected to 3 . The applied voltage $\mathrm{V}_{0}$ at the top electrode is $7 \mathrm{~V}$ and the voltage potentials at the measuring electrode $\mathrm{V}_{1}$ are set at $0.7 \mathrm{~V}$ for the case of air filled gap and $1.14 \mathrm{~V}$ for the case with inserted specimen. To study the influences of different model parameters, changes according the following eight scenarios are made in the model and their corresponding normalized corrections are calculated and shown in Fig. 3.
A. Reference model (No change);
B. Voltage potentials on the electrodes are $\mathrm{V}_{0}=7 \mathrm{~V}$, $\mathrm{V}_{1}=1.14 \mathrm{~V}$ for both the cases;
C. Shielding box removed (set to zero charge);
D. Size of the shielding box extends by $10 \%$;
E. Size of the shielding box reduced by $20 \%$;
F. No supporting material;
G. Size of the support reduced by $10 \%$;
$\mathrm{H}$. Add a cable with ground potential nearby.

Fig. 3 indicates that the measuring electrode voltage and the presence of a shielding box are the two most influential parameters. Thus, if the electrode voltage is not specified, the differences in the two capacitances will not be fully reproduced and therefore a smaller correction factor is obtained. If the shielding box is removed, the capacitive coupling between the two electrodes will be enhanced as there is no barrier to

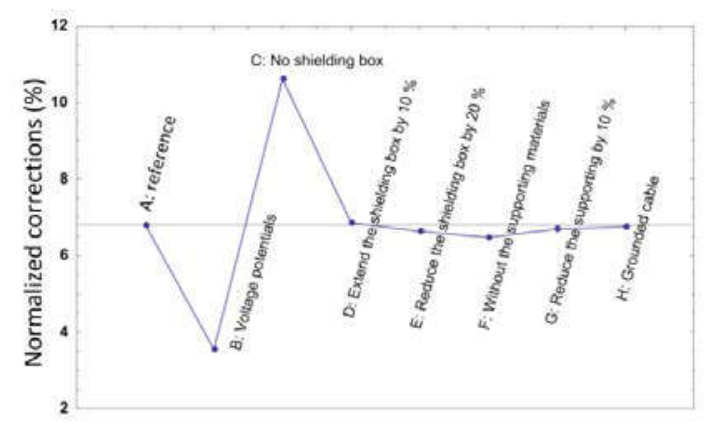

Fig. 3 - Normalized correction factor (\%) for eight scenarios considered in the model.

block the electric field lines from connecting the backsides of the electrodes. As this gives an increased capacitance, a larger correction is obtained. The other five scenarios have only slight impacts $(\max 0.4 \%)$ on the correction factor. Finally, one should note that almost $7 \%$ permittivity correction is required in general.

\section{Results and discussion}

Two specimens, PET film and polycarbonate, are employed to exemplify the correction of geometric influence using a fixed test gap and fixed specimen dimension, respectively.

\subsection{Fixed test gap}

Fig. 4 shows an estimated geometric correction function for a fixed $1 \mathrm{~mm}$ test gap (the electrode arrangement from Fig. 2). The plot indicates that with higher specimen permittivity, a larger correction is required. This is a result of a higher voltage on the measuring electrode and consequently larger influences from the surroundings. Further, the larger permittivity difference between air and the specimen enhance the field distortion at the electrode edge. The plot also shows that, for a constant specimen permittivity, a thin specimen requires more geometric correction than thicker ones. This results from a larger field distortion between the electrodes for samples filling only half the gap.

The obtained geometric correction function was applied for permittivity determination of several layers of PET films by the air reference method. The measurements were performed at $1 \mathrm{kHz}$ and $7 \mathrm{~V}$ voltage applied. Each film specimen was about $0.1 \mathrm{~mm}$ thick.

The measured and corrected permittivities for different number of layers are shown in Fig. 5. In additional, error estimations were also carried out for both results. For the measured ones, the distance measurement errors as well as the electrical measurement noise and drift errors were considered according to the method discussed in [3], whereas, in the corrected results, an additional error of $\pm 0.4 \%$ due to the geometric correction factor was added.

As show in Fig. 5, all the corrected results are about 7\% larger than the measured permittivity values. The corrected results are expected to be constant, however a slope is still observed with increasing number of 


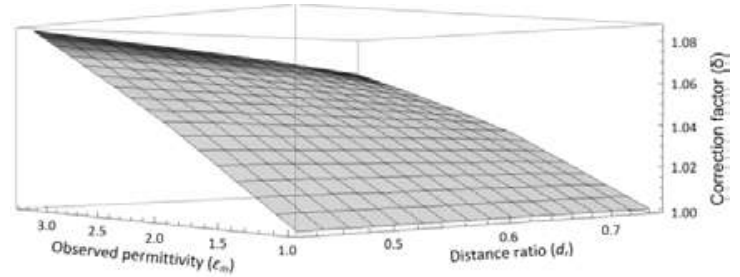

Fig. 4 - Geometric correction factors $(\delta)$ for a $1 \mathrm{~mm}$ fixed test gap with respect to the observed permittivity $\left(\varepsilon_{m}\right)$ and distance ratio $\left(d_{r}\right)$.

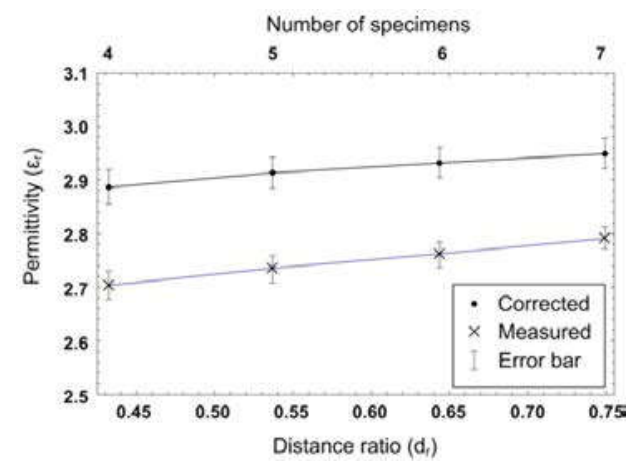

Fig. 5 - Measured and corrected permittivity of several layers of PET films (about $0.1 \mathrm{~mm}$ each) in $1 \mathrm{~mm}$ fixed test gap, the applied voltage for all the measurements were $1 \mathrm{kHz}$ and $7 \mathrm{~V}$.

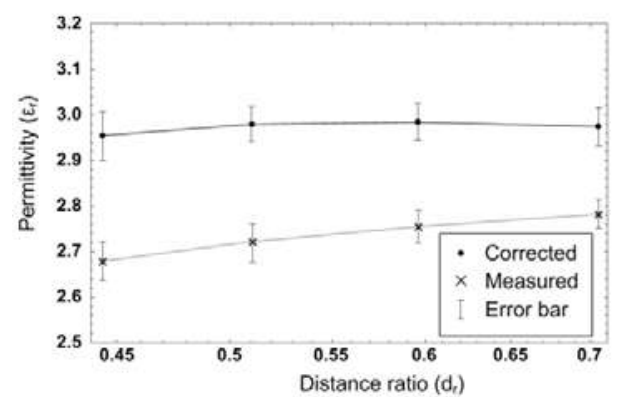

Fig. 6 - Measured and corrected permittivity of a $0.75 \mathrm{~mm}$ polycarbonate sample in four different test gaps, similar to Fig. 5

specimens, though the slope is smaller than the slope of the directly measured results and it is within half of the estimated error range. With increasing number of layers, a higher permittivity is obtained. This might be due to increased number of interfaces in the specimen. Another reason could be limitations forced by the $2 \mathrm{D}$ approach. From the sensitivity estimate in Fig. 5, we estimate that this is the best accuracy one reasonably can achieve with moderately complicated 2D calculations.

\subsection{Fixed specimen dimension}

As the number of interfaces may have a real effect on the permittivity, a homogenous sample was additionally studied at varying gap distances. The sample was 0.75 $\mathrm{mm}$ thick and made of polycarbonate. It was measured under four different gap distances and the corresponding correction factors were calculated from the model. The obtained correction factors for this case are similar to the ones shown in Fig. 4, due to space limitations, they cannot be shown here. The measured and the corrected permittivity of the polycarbonate sample are show in
Fig. 6. All the corrections for difference gaps are roughly about $7 \%$. The results indicate that the increasing slope of permittivity in the measured results is eliminated with the correction factors and a rather constant value of permittivity is observed. This indicates that the number of interfaces may be a cause of the remaining slope in figure 5 .

\section{Summary}

In dielectric material characterization, permittivities are often determined from a two-electrode fixture and subject to electrode geometric influences, if a nonnegligible voltage is generated at the measuring electrode over a high impedance current shunt, which is often desired in high voltage applications that requires high frequencies.

The geometric correction derived from finite element model calculations provides one possible way to increase the accuracy of permittivity by an order of magnitude, provided that the voltage on the measurement electrode is accounted for.

\section{Acknowledgment}

This work has been carried out within ELEKTRA project 36085 financed jointly by the Swedish Energy Agency, Elforsk and ABB.

\section{References}

[1] W. S. Zaengl, "Applications of dielectric spectroscopy in time and frequency domain for HV power equipment," Electrical Insulation Magazine, IEEE, vol. 19, pp. 9-22, 2003.

[2] B. Sonerud, "Characterization of Electrical Insulation Exposed to Arbitrary Voltage Waveforms," Doctoral Thesis, Department of materials and manufacturing technology, Chalmers University of Technology, Göteborg, 2010.

[3] X. Xu, T. Bengtsson, J. Blennow, and S. M. Gubanski, "Enhanced Accuracy in Dielectric Response Material Characterization by Air Reference Method," accepted by IEEE Trans. Dielectr. Electr. Insul., 2013.

[4] A. C. Lynch, "Edge capacitance in the measurement of dielectric properties," Electrical Engineers, Proceedings of the Institution of, vol. 120, pp. 934-938, 1973.

[5] L. Hartshorn, W. H. Ward, B. A. Sharpe, and B. J. O'Kane, "The effects of electrodes on measurements of permittivity and power factor on insulating materials in sheet form," Electrical Engineers, Journal of the Institution of, vol. 75, pp. 730-736, 1934.

[6] E.T.Hoch, "Electrode effects in the measurement of power factor and dielectric constant of sheet insulating materials," Bell System Tech. Jour., vol. 5, pp. 555572c, Oct. 1926.

[7] D. G. W. Goad and H. J. Wintle, "Capacitance corrections for guard gaps," Meas. Sci. Technol., vol. 1, p. $965,1990$.

[8] D. E. Woolley, "Edge Correction in Calculation of Dielectric Constant," Journal of Testing and Evaluation, vol. 39, pp. 140-149, Mar 2011.

[9] Comsol, AC/DC Module user's guide, Version 4.2a ed., 2011. 